\title{
BRANCHED COVERINGS. II
}

BY

R. E. STONG

\begin{abstract}
This paper improves the analysis of the possible cobordism classes $[M]-(\operatorname{deg} \phi)[N]$ for $\phi: M \rightarrow N$ a smooth branched covering of closed oriented smooth manifolds. It is assumed that the branch set is a codimension 2 submanifold.
\end{abstract}

1. Introduction. The purpose of this note is to prove

Proposition. Let $\phi: M^{n} \rightarrow N^{n}$ be a smooth branched covering of closed smooth oriented manifolds, with $n \equiv 0$ modulo $2(p-1)$, where $p$ is an odd prime. Let $\alpha=\left[M^{n}\right]-(\operatorname{deg} \phi)\left[N^{n}\right] \in \Omega_{n}$. Then

$$
s_{\left(i_{1(p-1) / 2}, \ldots, i_{r(p-1) / 2}\right)}(\mathfrak{p})[\alpha] \equiv 0 \quad \text { modulo } p .
$$

Further, these are the only conditions on a class $\alpha$ in $\Omega_{*}$ in order that it arise from a branched covering.

Note. These Pontrjagin numbers are precisely the ones which arise homotopy theoretically, i.e. using the mod $p$ Steenrod algebra.

This extends the results of [S], and should properly have been part of that paper. This material was not, however, obtained until after the manuscript was completed. Being based upon a different line of reasoning, its insertion would have required extensive revision.

2. The relations. Let $p$ be a fixed odd prime and let $q=(p-1) / 2$. Following $\mathrm{Wu}$ [W] one defines classes $v_{i} \in H^{4 i q}\left(M^{n} ; Z_{p}\right)$ in the $\bmod p$ cohomology of a closed oriented manifold by the formula

$$
\left\langle v_{i} \cup x,\left[M^{n}\right]\right\rangle=\left\langle\mathscr{P}^{i} x,\left[M^{n}\right]\right\rangle
$$

for all $x \in H^{n-4 i q}\left(M^{n} ; Z_{p}\right)$. One then lets $Q_{i}=\Sigma_{j}{ }^{P^{i-j}} v_{j} \in H^{4 i q}\left(M^{n} ; Z_{p}\right)$ and $Q=1+Q_{1}+Q_{2}+\cdots$, and has

LEMMA. If the Pontrjagin class of $M^{n}$ is written formally as

$$
\mathfrak{p}=\prod_{i}\left(1+x_{i}^{2}\right),
$$

then

$$
Q=\prod_{i}\left(1+x_{i}^{p-1}\right) \in H^{*}\left(M^{n} ; Z_{p}\right)
$$

Received by the editors November 19, 1981 and, in revised form, March 23, 1982.

1980 Mathematics Subject Classification. Primary 57R75, 55R55.

${ }^{1}$ The author is indebted to the National Science Foundation for financial support. 
Further, if $\bar{Q}=1 / Q$ is the dual class, then

$$
\left\langle\bar{Q}_{i} \cup x,\left[M^{n}\right]\right\rangle=\left\langle\chi\left(\mathscr{P}^{i}\right) x,\left[M^{n}\right]\right\rangle
$$

for all $x \in H^{n-4 i q}\left(M^{n} ; Z_{p}\right)$, where $\chi$ is the canonical antiautomorphism of the Steenrod algebra.

Note. The description of $\bar{Q}$ is due to Adams [A].

Being given $\phi: M^{n} \rightarrow N^{n}$ a branched covering of closed oriented manifolds, one has

$$
\mathfrak{p}(M)=\phi^{*}(\mathfrak{p}(N))\left\{1+\sum_{k} \sum_{l=1}^{\infty}(-1)^{l}\left(k^{2}-1\right) k^{2 l-2} \mathfrak{p}_{1, k}^{l}\right\}
$$

in integral cohomology (with equality modulo 2-torsion since the Whitney sum formula is used), by Brand's formula (see [S, §3]).

LEMMA.

$$
Q(M)=\phi^{*} Q(N) \cdot\left\{1+\sum_{k \equiv O(p)} \mathfrak{p}_{1, k}^{q}\right\}
$$

and

$$
\bar{Q}(M)=\phi^{*} \bar{Q}(N) \cdot\left\{\sum_{t=0}^{\infty}(-1)^{t} \sum_{k \equiv O(p)} p_{1, k}^{q t}\right\} .
$$

Proof. The class $\mathfrak{p}_{1, k}$ is induced by a map $M \rightarrow M O_{2}$, where $\mathfrak{p}_{1, \mathrm{k}}$ pulls back from the class going to $\mathfrak{p}_{1} \in \mathrm{H}^{2}\left(\mathrm{BO}_{2} ; Z\right)$ by the inclusion $B \mathrm{O}_{2} \rightarrow M \mathrm{O}_{2}$. The Pontrjagin class formula arose from $\mathfrak{p}\left(\gamma_{2}-\mu_{k} \gamma_{2}\right)=\left(1+x^{2}\right) /\left(1+k^{2} x^{2}\right)$. Correspondingly, $Q\left(\gamma_{2}-\mu_{k} \gamma_{2}\right)=\left(1+x^{p-1}\right) /\left(1+k^{p-1} x^{p-1}\right)$ which is 1 for $k \neq O(p)$ and which is $1+x^{p-1}$ for $k \equiv O(p)$. This gives the formula for $Q(M)$. To obtain that for $\bar{Q}$, one inverts, noting that $\left.\left\{\Sigma_{k \equiv O(p)} \mathfrak{p}_{l, k}^{q}\right\}\right]^{t}=\Sigma_{k \equiv O(p)} \mathfrak{p}_{1, k}^{q t}$.

LEMMA. The class $\Sigma_{k \equiv O(p)} \mathfrak{p}_{1, k}^{q t}$ belongs to the annihilator of the image of

$$
\phi^{*}: H^{*}\left(N ; Z_{p}\right) \rightarrow H^{*}\left(M ; Z_{p}\right) \text {. }
$$

Proof. For any $x \in H^{*}\left(N ; Z_{p}\right)$, one has

$$
\begin{aligned}
\left\langle\bar{Q}_{i}(M) \phi^{*} x,[M]\right\rangle & =\left\langle\chi\left(\mathscr{P}^{i}\right) \phi^{*} x,[M]\right\rangle=\left\langle\phi^{*}\left(\chi\left(\mathscr{P}^{i}\right) x\right),[M]\right\rangle \\
& =\left\langle\phi^{*}\left(\bar{Q}_{i}(N) x\right),[M]\right\rangle=\left\langle\phi^{*}\left(\bar{Q}_{i}(N)\right) \cdot \phi^{*} x,[M]\right\rangle,
\end{aligned}
$$

and so $\bar{Q}_{i}(M)-\phi^{*} Q_{i}(N) \in\left(\operatorname{im} \phi^{*}\right)^{\perp}$, and $\bar{Q}(M)-\phi^{*} \bar{Q}(N) \in\left(\operatorname{im} \phi^{*}\right)^{\perp}$.

Let $Z=\sum_{k \equiv 0} \mathfrak{p}_{1, k}^{q}$, and then for any $y \in H^{*}\left(N ; Z_{p}\right)$,

$$
\begin{aligned}
0 & =\left\langle\left\{\bar{Q}(M)-\phi^{*} \bar{Q}(N)\right\} \phi^{*}(Q(N) y),[M]\right\rangle \\
& =\left\langle\left\{\left(1-Z+Z^{2}+\cdots\right)-1\right\} \phi^{*} \bar{Q}(N) \phi^{*}(Q(N) y),[M]\right\rangle \\
& =\left\langle\left\{-Z+Z^{2}-Z^{3}+\cdots\right\} \phi^{*}(y),[M]\right\rangle,
\end{aligned}
$$

and so $Z^{t}=\sum_{k \equiv 0} \mathfrak{p}_{1, k}^{q^{t}} \in\left(\operatorname{im} \phi^{*}\right)^{\perp}$ for all $t \geqslant 1$.

COROllary. If $n \equiv 0 \bmod 2(p-1)$, and $\omega$ is any partition of $n / 2(p-1)$, then $Q_{\omega}[\alpha]=0$. 
Proof. For $Z=\Sigma_{k \equiv 0} \mathfrak{p}_{1, k}^{q}$, one has $Q(M)=\phi^{*} Q(N)(1+Z)$ and so $Q_{i}(M)=$ $\phi^{*} Q_{i}(N)+\phi^{*} Q_{i-1}(N) \cdot Z$. Expanding $Q_{\omega}(M)$ one then has $Q_{\omega}(M)=\phi^{*} Q_{\omega}(N)+$ $\sum_{s>0} Z^{s} \cdot \phi^{*} Q_{\omega, s}(N)$, where $Q_{\omega, s}(N)$ is the appropriate polynomial in $Q_{i}(N)$ depending on $\omega$ and $s$. Evaluating on $[M], Q_{\omega}[M]=\phi^{*} Q_{\omega}(N)[M]=(\operatorname{deg} \phi) Q_{\omega}[N]$, since $Z^{s} \phi^{*} Q_{\omega, s}(N)[M]=0$.

Since the $Q_{\omega}$ and $s_{\left(i, q, \ldots, i_{r} q\right)}(\mathfrak{p})$ are simply two different bases for the homotopical Pontrjagin classes, one has the relations given in the Proposition.

One then has

Proposition. The set of possible s-numbers $s_{m}(\mathfrak{p})\left[\left[M^{4 m}\right]-d\left[N^{4 m}\right]\right]$ for $d$-fold branched coverings of closed oriented manifolds is the subgroup $s_{m}^{d} Z$ of the integers where

$$
s_{m}^{d}=a \cdot \operatorname{gcd}\left\{\left(1-2^{2 m}\right),\left(1-3^{2 m}\right), \ldots,\left(1-d^{2 m}\right)\right\}
$$

and $a=p_{1} p_{2} \cdots p_{r}$ is the product of the odd primes $p_{i}$ with $p_{i} \leqslant d$ and $p_{i}-1$ dividing $2 m$.

Proof. This is the result of [S, §4], including the new fact that

$$
s_{m}(\mathfrak{p})\left[\left[M^{4 m}\right]-d\left[N^{4 m}\right]\right]
$$

is divisible by $p$ if $p-1$ divides $2 m$.

3. Completeness. To verify that no other relations occur is largely a case of combining known results. For this, let $B_{n} \subset \Omega_{n}$ be the set of classes $\alpha=\left[M^{n}\right]-$ $(\operatorname{deg} \phi)\left[N^{n}\right]$ coming from branched covers. It is immediate that $B_{*} \subset \Omega_{*}$ is then an ideal $\left(\Omega_{*}\right.$-submodule).

By Proposition 4 of [S], $B_{n} \subset \Omega_{n}$ is a subgroup of odd index. Thus $B_{*}$ contains Tor $\Omega_{*}$ and further one need only consider $\Omega_{*} / B_{*}$ for each odd prime $p$.

From the previous proposition (taking $d$ large), one has classes $y_{4 i} \in B_{4 i}$ so that $s_{i}(\mathfrak{p})\left[y_{4 i}\right]$ is the product of those odd primes $p$ with $p-1$ dividing $2 i$, and $\Omega_{*} /$ Tor $\Omega_{*}$ is the integral polynomial ring on generators $x_{4 i}$ characterized by $s_{i}(\mathfrak{p})\left[x_{4 i}\right]=1$ if $2 i \neq p^{s}-1$ and $s_{i}(\mathfrak{p})\left[x_{4 i}\right]=p$ if $2 i=p^{s}-1$.

Fixing an odd prime $p$, let $x_{4 i}^{\prime}=x_{4 i}$ if $2 i$ is not divisible by $p-1$ or is $2 i=p^{s}-1$, and let $x_{4 i}^{\prime}=p x_{4 i}$ otherwise. Let $B_{*}^{\prime} \subset \Omega_{*} /$ Tor $\Omega_{*}$ be the ideal generated by the $x_{4 i}^{\prime}$. Then $\Omega_{*} /\left\{\right.$ Tor $\left.\Omega_{*}+B_{*}^{\prime}\right\}$ is isomorphic to the $Z_{p}$ polynomial ring on the classes $x_{4 i}$ with $2 i \equiv 0(p-1), 2 i \neq p^{s}-1$, and its dual is the space of Pontrjagin numbers $(\bmod p)$

$$
s_{\left(i_{1} q, \ldots, i_{r} q\right)}(\mathfrak{p})
$$

where $q=(p-1) / 2$ and $i_{\alpha} q \neq\left(p^{s}-1\right) / 2$. Thus $B_{*} \subset$ Tor $\Omega_{*}+B_{*}^{\prime}$. Since $s_{i}\left[y_{4 i}\right]$ $=\lambda_{i} s_{i}\left[x_{4 i}^{\prime}\right]$, with $\lambda_{i}$ relatively prime to $p$, for each $i, B_{*} \subset$ Tor $\Omega_{*}+B_{*}^{\prime}$ must have index relatively prime to $p$ in each dimension; i.e. the matrices $s_{\omega}\left[y_{4 \omega^{\prime}}\right]$ and $s_{\omega}\left[x_{4 \omega^{\prime}}^{\prime}\right]$ with $\omega, \omega^{\prime}$ partitions of $m$ are both triangular when ordered by refinement with diagonal entries being divisible by the same powers of $p$, and hence the indices of $B_{*}$ and Tor $\Omega_{*}+B_{*}^{\prime}$ in $\Omega_{*}$ are divisible by the same power of $p$.

Since this holds for each odd prime, $B_{*} \subset \Omega_{*}$ must be precisely the set of classes which satisfy the relations of the Proposition. 


\section{4. $d$-fold covers.}

OBSERVATION. The set of classes in $\Omega_{*} /$ Tor $\Omega_{*}$ arising from branchings is the ideal generated by the classes of the branchings $\phi: Q_{k}^{2 r} \rightarrow \mathbf{C} P^{2 r}$ of the $k$-drics over the complex projective spaces (and $\frac{1}{2}$ the class for the quadric; $k=2$ ).

This is an immediate consequence of the calculation of $s_{m}^{d}$, for the $k$-drics gave the upper bound.

For branchings of a fixed degree, these classes may not generally be sufficient. One would like to know $B_{*}^{d} \subset \Omega_{*}$, the set of classes arising from branchings of degree $d$.

OBSERVATION. For 2-fold covers, $B_{*}^{2} \subset \Omega_{*}$ is the ideal generated by Tor $\Omega_{*}$ and the classes $\frac{1}{2}\left\{\left[Q_{1}^{2 r}\right]-2\left[\mathbf{C} P^{2 r}\right]\right\}=\left[H P^{r}\right]-\left[\mathbf{C} P^{2 r}\right]$.

Proof. According to Hattori [H, Lemma 3.2], if $\alpha \in B_{*}^{2}$, then $2 \alpha$ is realized by a 2-fold branching with oriented branch set. Such branchings are classified by $D(\gamma \otimes \gamma) \simeq B S O_{2}$, and the standard base over $\Omega_{*}$ of $\Omega_{*}\left(B S O_{2}\right)$ is given by the inclusions $\mathrm{CP} \rightarrow \mathrm{BSO}_{2}=\mathrm{CP}$, corresponding to the branchings of the quadrics. By Proposition 4 of [S], $B_{*}^{2} \subset \Omega_{*}$ has odd index, and the assertion follows.

OBSERVATION. Hattori's result [H, Lemma 3.2] is true for all branched coverings. Given $\phi: M^{n} \rightarrow N^{n}$ a branched covering of oriented manifolds there is a branched covering $\phi: \tilde{M}^{n} \rightarrow \tilde{N}^{n}$ of oriented manifolds with the same degree for which one has

(1) a commutative diagram

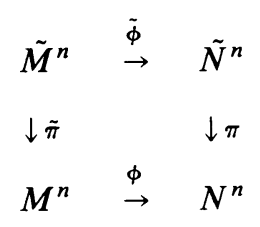

in which $\pi, \pi^{\prime}$ are 2-fold branched coverings, with $B_{\pi}$ and $B_{\phi}$ disjoint,

(2) $B_{\tilde{\phi}}$ has oriented branch set, specifically the orientation cover of $B_{\phi}$, and

(3) $\left[\tilde{M}^{n}\right]=2\left[M^{n}\right],\left[\tilde{N}^{n}\right]=2\left[N^{n}\right]$ in $\Omega_{*}$.

Proof. Let $C_{\phi} \subset B_{\phi}$ be the submanifold dual to $w_{1}$, and then $\phi^{-1} C_{\phi} \subset \phi^{-1} B_{\phi}$ is also dual to $w_{1}$. Hattori constructs a 2-fold branching with branch set the inverse image of $C_{\phi}$ in the sphere of $\nu$ over $B_{\phi}$, to give $\pi: \tilde{N}^{n} \rightarrow N^{n}$, by what he calls the Dold construction, with $\pi^{-1} B_{\phi} \rightarrow B_{\phi}$ being the orientation cover. To obtain $\tilde{\pi}$ one pulls $\pi$ back over $\phi$. Because $\phi^{-1} C_{q}$ is also dual to $w_{1}, \tilde{M}^{n}$ is obtained from $M^{n}$ by the same construction.

Note. The submanifold of $S(\nu)$ over $C_{\phi}$ has normal bundle $l+1$, with $l$ a line bundle, giving a map $N^{n} \rightarrow M\left(\gamma_{1} \oplus 1\right)$ where $\gamma_{1}$ is the line bundle over $B O_{1}$. Since $\mu_{2}\left(\gamma_{1} \oplus 1\right) \cong \gamma_{1} \oplus 1$, this map may be composed with $M\left(\gamma_{i} \oplus 1\right) \rightarrow M\left(\mu_{2} \gamma_{2}\right) \rightarrow$ $M\left(\gamma_{2} \oplus \mu_{2} \gamma_{2}\right)$, where the latter classifies 2-fold covers, giving rise to a 2 -fold branching with the given branch set. Since $M\left(\gamma_{1} \oplus 1\right) \cong \Sigma R P^{\infty}$ has trivial rational cohomology, $\pi: \tilde{N}^{n} \rightarrow N^{n}$ has $\pi^{*} \mathfrak{p}(N)=\mathfrak{p}(\tilde{N})$ rationally and $\left[\tilde{N}^{n}\right]=2\left[N^{n}\right]$ in $\Omega_{*} /$ Tor $\Omega_{*}$. By observing that $\phi^{-1} B_{\phi}$ is the orientation cover of $B_{\phi}$, as Hattori does, $\left[\tilde{N}^{n}\right]=0$ in $\Re_{*}$, so $\left[\tilde{N}^{n}\right]=2\left[N^{n}\right]$ in $\Omega_{*}$. This latter point is the only one that is not general nonsense, and depends on the choice of the branch set for $\pi$. 
Note. Hattori constructs $\tilde{X}$ over $X$, the total space of the branched cover, and uses the involution to form a quotient. (His "Dold construction" is not performed over the corresponding space in the diagram of 1.)

OBSERVATION. For $n \leqslant 32, B_{n}^{3}=B_{n}$ and every class which can be obtained from a branched cover actually arises from a 3-fold cover. In particular, in small dimensions $B_{*}^{3}$ is generated as ideal by the classes of the 3-drics, half the classes of the quadrics, and Tor $\Omega_{*}$.

Proof. One checks that $s_{m}^{3}=\lim _{d} s_{m}^{d}$ for $m \leqslant 8$. Since $B_{*}$ is the ideal generated by its indecomposables, it coincides with $B_{*}^{3}$ through the given range.

Note. One should calculate $s_{m}^{3}$ through a larger range, but I am too lazy. This illustrates the situation adequately.

\section{Remarks.}

ReMARK 1. For the prime 3, $q=1$. Thus all mod 3 Pontrjagin numbers vanish for the classes $\alpha$ coming from branchings.

REMARK 2. For the prime 2, the argument breaks down completely because of failure of the Whitney sum formula for Pontrjagin classes. The analogous statement occcurs when one assumes the branch set $B_{\phi}$ is orientable, and then all Pontrjagin numbers are even, and that is a very close analogue.

Remark 3. The proof of Proposition 5 of [S], which could be superceded by the slick formalism of this proof, probably gives more insight into the relations. The divisibilities occur because local branchings give nontrivial classes in $\Omega_{*}\left(B \Sigma_{d}\right)$. Unfortunately, calculation with $\Omega_{*}\left(B \Sigma_{d}\right)$ is impossibly hard, and one can only carry the argument through for $d<2 p$.

\section{REFERENCES}

[A] J. F. Adams, On formulae of Thom and Wu, Proc. London Math. Soc. 11 (1961), 741-752.

[H] A. Hattori, Genera of ramified coverings, Math. Ann. 195 (1972), 208-226.

[S] R. E. Stong, Branched coverings. I, Trans. Amer. Math. Soc. 276 (1983), 375-402.

[W] Wen-Tsün Wu, On Pontrjagin classes. II, Acta Math. Sinica 4 (1954), 171-199. 